\title{
STABILITY OF PICARD SHEAVES FOR VECTOR BUNDLES ON CURVES
}

\author{
GEORG HEIN AND DAVID PLOOG
}

\begin{abstract}
We show that for any stable sheaf $E$ of slope $\mu(E)>2 g-1$ on a smooth, projective curve of genus $g$, the associated Picard sheaf $\hat{E}$ on the Picard variety of the curve is stable. We introduce a homological tool for testing semistability of Picard sheaves.
\end{abstract}

\section{INTRODUCTION}

Throughout, $X$ is a smooth, projective genus $g$ curve over an algebraically closed field $k$. Let Pic $:=\operatorname{Pic}^{0}(X)$ be the Picard variety of $X$ and $\mathcal{P}$ the Poincaré line bundle on $X \times$ Pic.

For a vector bundle $E \in \operatorname{Coh}(X)$, its Picard complex is the Fourier-Mukai (or integral) transform $\hat{E}:=\mathrm{FM}_{\mathcal{P}}(E)$, an object of $\mathrm{D}^{\mathrm{b}}(\mathrm{Pic})$. We denote its two cohomology sheaves by $\hat{E}^{0}$ and $\hat{E}^{1}$ and call these the Picard sheaves of $E$. Our goal is to show that $\hat{E}$ is (semi)stable on Pic for general, (semi)stable bundles $E$ on $X$ for certain slopes. In fact, we prove this by showing that $\hat{E}$ is semistable when restricted to curves $i$ : $X \hookrightarrow$ Pic. We have the following result; see Corollaries 1.11, 2.3, 3.1, and 3.4:

Theorem A. If $E$ is a stable bundle on $X$ of slope $\mu(E)>2 g-1$, then the Picard sheaf $\hat{E}^{0}$ is stable on Pic. Dually, if $E$ is stable of slope $\mu(E)<-1$, then the Picard sheaf $\hat{E}^{1}$ is stable. The analogous statements hold for semistability, using the non-strict inequalities.

Using our concept of orthogonality, we obtain results for Picard sheaves for generic semistable bundles of slope $\mu \in[g-2, g]$ unless $\mu=g-1$; see Proposition 3.7 and Corollary 3.8.

Theorem B. For $\mu \in(g-1, g]$, there exists a semistable bundle $E$ on $X$ of slope $\mu$ such that its Picard sheaf $\hat{E}^{0}$ is semistable. Dually, for $\mu \in[g-2, g-1)$, there exists a semistable bundle $E$ on $X$ of slope $\mu$ such that its Picard sheaf $\hat{E}^{1}$ is semistable.

In order to show Theorem A, we generalise Clifford's theorem about estimating global sections, from divisors to not necessarily semistable vector bundles. If $E=L_{1} \oplus \cdots \oplus L_{r}$ is a direct sum of line bundles with all $\operatorname{deg}\left(L_{i}\right) \in[0,2 g-2]$, then $h^{0}\left(L_{i}\right)-1 \leq \operatorname{deg}\left(L_{i}\right) / 2$ by the classical Clifford theorem. This sums up to $h^{0}(E)-r \leq \operatorname{deg}(E) / 2$. Therefore, the best generalisation one can hope for is the following result; see Proposition 4.1, where we also give precise information about the equality case.

Proposition C. Let $E$ be a vector bundle of rank $r$ and degree $d$ on the smooth projective curve $X$ of genus $g$. If $\mu_{\max }(E) \leq 2 g-2$ and $\mu_{\min }(E) \geq 0$, then we have the estimate

$$
h^{0}(E)-r \leq \frac{d}{2} \text {. }
$$

The special case of semistable vector bundles of slope $\mu \in[0,2 g-2]$ was already proved in [2, Theorem 2.1]. If the slope of a semistable bundle $E$ is not in this interval, then either $H^{0}(E)=0$ or $H^{1}(E)=0$, and the dimension of the remaining cohomology group is computed by the Riemann-Roch theorem.

MSC 2010: 14H60; 14F05 
Known results. Classically, Picard sheaves are pushforwards of Poincaré bundles along the projection $X \times \operatorname{Pic}^{d}(X) \rightarrow \operatorname{Pic}^{d}(X)$, see [8, 12,9]. Later on, this notion was extended to pushforwards of universal bundles onto the moduli space.

Kempf [6] has shown that the Picard bundle on $\operatorname{Pic}^{d}(X)$ is stable for $d=2 g-1$, the smallest degree where the Picard complex $\hat{\mathcal{O}}_{X}=\left(\hat{\mathcal{O}}_{X}\right)^{0}$ is a vector bundle. Here and later on, stability on the Picard variety is meant with respect to the polarisation by the theta divisor. In [3], Ein and Lazarsfeld proved the stability of Picard bundles on $\operatorname{Pic}^{d}(X)$ for $d \geq 2 g$. They do this by restricting the Picard bundles to the canonical curves $X \subset \operatorname{Pic}^{d}(X)$ and $(-X) \subset \operatorname{Pic}^{d}(X)$.

$\mathrm{Li}$ [7] considers the moduli space $\mathscr{M}_{r, d}$ of stable bundles on $X$ of rank $r$ and degree $d$ with $d>2 r(g-1)$ and $(d, r)=1$. If $\mathscr{U}$ denotes the universal bundle on $X \times \mathscr{M}_{r, d}$, then Li shows that the Picard bundle $\operatorname{pr}_{2 *} \mathscr{U}$ on $\mathscr{M}_{r, d}$ is stable if $d>2 g r$.

In [1], the authors consider the same question for the moduli space of stable bundles of rank $r$ and fixed determinant $L$. Then the associated Picard bundle on the moduli space is stable (with respect to the unique polarisation) if $\operatorname{deg}(L)>2 r(g-1)$ and $(r, \operatorname{deg}(L))=1$.

Here, we go back to the case of Jacobians, but now we consider the Poincare bundle twisted by a vector bundle pulled back from the curve. In other words, we study preservation of (semi)stability for the Fourier-Mukai transform along the Poincaré bundle, taking bundles on the curve to sheaves on its Picard variety.

Acknowledgments. This work has been supported by SFB/TR 45 "Periods, moduli spaces and arithmetic of algebraic varieties".

Conventions. $X$ is always a smooth, projective curve of genus $g$ over a fixed algebraically closed field. The slope of a vector bundle is denoted $\mu(E)=\operatorname{deg}(E) / \operatorname{rk}(E)$. Note $\mu(E \otimes F)=\mu(E)+\mu(F)$. We repeatedly use the Riemann-Roch formula, always in the form $\chi(E)=\operatorname{rk}(E)(\mu(E)+1-g)$. We write $h^{0}(E)=\operatorname{dim} H^{0}(E)$ and $h^{1}(E)=\operatorname{dim} H^{1}(E)$. We denote projections by $\mathrm{pr}_{X}, \mathrm{pr}_{Y}: X \times Y \rightarrow X$ or by $\mathrm{pr}_{1}, \mathrm{pr}_{2}: X \times X \rightarrow X$. Given sheaves $E$ and $F$ on $X$, then as usual we write $E \otimes F=\operatorname{pr}_{1}^{*} E \otimes \operatorname{pr}_{2}^{*} F$. Sometimes, we follow standard usage and pack two statements into one, using (semi)stability and $(\leq)$.

\section{ORthogonality AND STABILITY}

1.1. Definition of orthogonality and first properties. We first recall a classical result of Faltings [4, Theorem 1.2], which expresses semistability of a vector bundle on a curve as an orthogonality condition on $X$ :

Theorem 1.1 (Faltings 1993). A vector bundle $E$ on $X$ is semistable if and only if there exists a vector bundle $0 \neq F$ such that $H^{*}(E \otimes F)=0$.

Proof. For the convenience of reader, we prove the easy implication of this statement. Assume $H^{*}(E \otimes F)=0$, and let $0 \neq G \subset E$ be a destabilising subsheaf, i.e. $\mu(G)>\mu(E)$.

Then $G \otimes F \subset E \otimes F$ with $\mu(G \otimes F)>\mu(E \otimes F)$. Since $H^{*}(E \otimes F)=0$, RiemannRoch gives $\chi(G \otimes F)>\chi(E \otimes F)=0$. Hence $h^{0}(G \otimes F)>0$, which contradicts $H^{0}(G \otimes F) \subseteq H^{0}(E \otimes F)=0$.

Here, we introduce two other orthogonality notions, on $X \times X$, which work well with Picard sheaves:

Definition. Two coherent sheaves $E$ and $F$ on $X$ are called orthogonal with respect to $-\Delta$ if the sheaf $\operatorname{pr}_{1}^{*} E \otimes \mathcal{O}_{X \times X}(-\Delta) \otimes \operatorname{pr}_{2}^{*} F$ on $X \times X$ has vanishing cohomology. 
Analogously, $E$ and $F$ are orthogonal with respect to $\Delta$ if the cohomology groups of $\operatorname{pr}_{1}^{*} E \otimes \mathcal{O}_{X \times X}(\Delta) \otimes \operatorname{pr}_{2}^{*} F$ vanish. In short, we write

$$
\begin{aligned}
& E \frac{1}{-\Delta} F \Longleftrightarrow H^{*}(E \otimes F(-\Delta))=0, \\
& E \frac{1}{\Delta} F \Longleftrightarrow H^{*}(E \otimes F(\Delta))=0 .
\end{aligned}
$$

Orthogonality has the following simple numerical description:

Lemma 1.2. Let $E$ and $F$ be coherent sheaves on $X$.

(i) If $H^{*}(E \otimes F)=0$, then $\mu(F)=-\mu(E)+g-1$.

(ii) If $E \frac{\perp}{-\Delta} F$, then $\mu(F)=g+\frac{g}{\mu(E)-g}$.

Proof. (i) follows readily from Riemann-Roch.

(ii) The cohomology of the exact sequence $\left.0 \rightarrow E \otimes F(-\Delta) \rightarrow E \otimes F \rightarrow E \otimes F\right|_{\Delta} \rightarrow 0$, using $\left.E \otimes F\right|_{\Delta} \cong E \otimes F$ and $H^{*}(E \otimes F(-\Delta))=0$ from $E_{-\Delta}^{\perp} F$ gives: $H^{*}(E \otimes F) \cong$ $H^{*}(E \otimes F) \cong H^{*}(E) \otimes H^{*}(F)$, the latter isomorphism from the Künneth formula. Hence $\chi(E \otimes F)=\chi(E) \chi(F)$, or $(\mu(E)+\mu(F)+1-g)=(\mu(E)+1-g)(\mu(F)+1-g)$ by Riemann-Roch. Manipulating this equation yields the claimed formula.

We collect the following statements for referability; the proofs are immediate:

Lemma 1.3. For vector bundles $E$ and $F$ on $X$, we have the three equivalences:

$$
\begin{aligned}
& E \frac{\perp}{-\Delta} F \Longleftrightarrow F \frac{\perp}{-\Delta} E, \\
& E \frac{\perp}{\Delta} F \Longleftrightarrow F \frac{1}{\Delta} E, \\
& E \frac{\perp}{-\Delta} F \Longleftrightarrow\left(\omega_{X} \otimes E^{\vee}\right) \frac{\perp}{\Delta}\left(\omega_{X} \otimes F^{\vee}\right) \quad \text { (Serre duality). }
\end{aligned}
$$

Definition. We define two functors $\mathrm{F}_{+}, \mathrm{F}_{-}: \operatorname{Coh}(X) \rightarrow \mathrm{D}^{\mathrm{b}}(X)$ by

$$
\begin{aligned}
& \mathrm{F}_{+}(E)=\operatorname{Rpr}_{2 *}\left(\mathcal{O}_{X \times X}(\Delta) \otimes \operatorname{pr}_{1}^{*} E\right), \\
& \mathrm{F}_{-}(E)=\operatorname{Rpr}_{2 *}\left(\mathcal{O}_{X \times X}(-\Delta) \otimes \operatorname{pr}_{1}^{*} E\right) .
\end{aligned}
$$

We denote the cohomology sheaves by

$$
\mathrm{F}_{+}^{i}=R^{i} \operatorname{pr}_{2 *}\left(\mathcal{O}_{X \times X}(\Delta) \otimes \operatorname{pr}_{1}^{*} E\right)
$$

and similarly for $\mathrm{F}_{-}^{i}(E)$. Since the fibres are 1-dimensional and objects in $\mathrm{D}^{\mathrm{b}}(X)$ decompose into their cohomology sheaves, we have $\mathrm{F}_{+}(E)=\mathrm{F}_{+}^{0}(E) \oplus \mathrm{F}_{+}^{1}(E)[-1]$, and $\mathrm{F}_{-}(E)=\mathrm{F}_{-}^{0}(E) \oplus \mathrm{F}_{-}^{1}(E)[-1]$.

Lemma 1.4. For vector bundles $E$ and $F$ on $X$, we have the two equivalences

(i) $\quad E \frac{\perp}{-\Delta} F \Longleftrightarrow H^{*}\left(\mathrm{~F}_{-}^{0}(E) \otimes F\right)=0$, and $H^{*}\left(\mathrm{~F}_{-}^{1}(E) \otimes F\right)=0 \quad$ and

$$
\text { (ii) } \quad E \frac{\perp}{\Delta} F \Longleftrightarrow H^{*}\left(\mathrm{~F}_{+}^{0}(E) \otimes F\right)=0 \text {, and } H^{*}\left(\mathrm{~F}_{+}^{1}(E) \otimes F\right)=0 \text {. }
$$

Proof. We only show (i), as the proof of (ii) works analogously.

Let $G=\operatorname{pr}_{1}^{*} E \otimes \mathcal{O}_{X \times X}(-\Delta) \otimes \operatorname{pr}_{2}^{*} F$. We compute the cohomology of $G$ using the Leray spectral sequence for $\mathrm{pr}_{2}$. For dimension reasons, the spectral sequence degenerates, thus

$$
H^{0}(G)=H^{0}\left(\operatorname{pr}_{2 *} G\right), \quad H^{2}(G)=H^{1}\left(R^{1} \operatorname{pr}_{2 *} G\right)
$$

and a short exact sequence

$$
0 \rightarrow H^{1}\left(\operatorname{pr}_{2 *} G\right) \rightarrow \underset{3}{H^{1}(G)} \rightarrow H^{0}\left(R^{1} \operatorname{pr}_{2 *} G\right) \rightarrow 0
$$


Therefore, we get $R^{i} \operatorname{pr}_{2 *}(G)=\mathrm{F}_{-}^{i}(E) \otimes F$, using the projection formula, together with the definitions of $G$ and $\mathrm{F}_{-}^{i}(E)$. This proves both implications of the assertion, noting that $E \underset{-\Delta}{\perp} F$ is tantamount to $H^{*}(G)=0$.

Proposition 1.5. For a coherent sheaf $E$ on $X$, the following conditions are equivalent:

(1) There exists a coherent $F \neq 0$ such that $E \frac{\perp}{-\Delta} F$.

(2) $\mathrm{F}_{-}^{0}(E)$ and $\mathrm{F}_{-}^{1}(E)$ are semistable sheaves of the same slope.

Similarly there exists such an equivalence for orthogonality with respect to $+\Delta$.

(1') There exists a coherent $F \neq 0$ such that $E \stackrel{\perp}{\perp} F$.

(2') $\mathrm{F}_{+}^{0}(E)$ and $\mathrm{F}_{+}^{1}(E)$ are semistable sheaves of the same slope.

Proof. We start with (1) $\Longrightarrow(2)$. Assume $E \frac{\perp}{-\Delta} F$ for some $F \neq 0$. From Lemma 1.4 we conclude $H^{*}\left(\mathrm{~F}_{-}^{i}(E) \otimes F\right)=0$ for $i \in\{0,1\}$. By the easy direction of Theorem 1.1, $\mathrm{F}_{-}^{i}(E)$ is semistable. Moreover, we get $\mu\left(\mathrm{F}_{-}^{i}(E)\right)=-\mu(F)+g-1$ from Lemma 1.2(i)

$(2) \Longrightarrow(1)$. With $\mathrm{F}_{-}^{0}(E)$ and $\mathrm{F}_{-}^{1}(E)$ semistable of the same slope, their direct sum $\mathrm{F}_{-}^{0}(E) \oplus \mathrm{F}_{-}^{1}(E)$ is semistable as well. Thus by Faltings' Theorem 1.1, there exists a sheaf $F \neq 0$ such that $H^{*}\left(\left(\mathrm{~F}_{-}^{0}(E) \oplus \mathrm{F}_{-}^{1}(E)\right) \otimes F\right)=0$. By Lemma 1.4 we are done.

Remark 1.6. Whenever we have an orthogonal pair $E \stackrel{\perp}{\perp} F$ with non-zero sheaves $E$ and $F$, then we conclude that the six sheaves $E, \mathrm{~F}_{-}^{0}(E), \mathrm{F}_{-}^{1}(E), F, \mathrm{~F}_{-}^{0}(F)$, and $\mathrm{F}_{-}^{1}(F)$ are semistable.

However, in most situations we consider here, one of the two sheaves $\mathrm{F}_{-}^{0}(E)$ or $\mathrm{F}_{-}^{1}(E)$ will be zero. Anyway, they cannot be both zero, as the following argument shows: Set $r=\operatorname{rk}(E), R=\operatorname{rk}\left(\mathrm{F}_{-}^{0}(E)\right)-\operatorname{rk}\left(\mathrm{F}_{-}^{1}(E)\right), d=\operatorname{deg}(E)$, and $D=\operatorname{deg}\left(\mathrm{F}_{-}^{0}(E)\right)-\operatorname{deg}\left(\mathrm{F}_{-}^{1}(E)\right)$. A short Riemann-Roch computation along the lines of Lemma 1.2(ii) gives

$$
\left(\begin{array}{l}
R \\
D
\end{array}\right)=\left(\begin{array}{rr}
-g & 1 \\
0 & -1
\end{array}\right)\left(\begin{array}{l}
r \\
d
\end{array}\right)
$$

Thus, we can deduce the pair $(r, d)$ from $(R, D)$ unless $g=0$.

1.2. Picard sheaves, and embedding $X$ into the Picard scheme. We denote by $\mathrm{Pic}=\mathrm{Pic}^{0}(X)$ the Picard scheme of the smooth curve $X$ and by $\mathcal{P}$ the Poincaré bundle on $X \times$ Pic. Fixing a point $P_{0} \in X(k)$, we normalise the Poincaré bundle by the additional assumption that $\left.\mathcal{P}\right|_{\left\{P_{0}\right\} \times \text { Pic }} \cong \mathcal{O}_{\text {Pic }}$. The projections from $X \times$ Pic will be denoted

$$
X \stackrel{\mathrm{pr}_{X}}{\stackrel{\mathrm{P}}{\leftrightarrows}} X \times \mathrm{Pic} \stackrel{\mathrm{pr}_{P}}{\longrightarrow} \text { Pic . }
$$

For a coherent sheaf $E$ on $X$, we define its Picard complex to be the object

$$
\hat{E}:=\mathrm{FM}_{\mathcal{P}}(E)=\operatorname{Rpr}_{P *}\left(\mathcal{P} \otimes \operatorname{pr}_{X}^{*} E\right)
$$

in the derived category $\mathrm{D}^{\mathrm{b}}(\mathrm{Pic})$. Since $\mathcal{P}$ is $\mathrm{pr}_{1}$-flat, and $\mathrm{pr}_{2}$ is of dimension 1 , we have only two cohomology sheaves of our complex, and we call these the Picard sheaves of $E$ :

$$
\begin{aligned}
& \hat{E}^{0}:=\mathrm{FM}_{\mathcal{P}}^{0}(E)=\operatorname{pr}_{P *}\left(\mathcal{P} \otimes \operatorname{pr}_{X}^{*} E\right), \quad \text { and } \\
& \hat{E}^{1}:=\mathrm{FM}_{\mathcal{P}}^{1}(E)=R^{1} \operatorname{pr}_{P *}\left(\mathcal{P} \otimes \operatorname{pr}_{X}^{*} E\right) .
\end{aligned}
$$

We are interested mainly in the case when one of $\hat{E}^{0}$ or $\hat{E}^{1}$ is zero. To study their semistability, we will restrict them to the curves $(X)_{M}$ and $(-X)_{N}$, to be defined next.

For any line bundle $M$ of degree 1, we have an embedding of $\iota_{M}: X \rightarrow$ Pic given by $P \mapsto M(-P)$. The image of $\iota_{M}$ is denoted by $(-X)_{M}$. In the same way, any line bundle $N$ of degree -1 defines an embedding $\iota_{N}: X \rightarrow$ Pic by $\iota_{N}(P)=N(P)$ with image $(X)_{N}$. The next proposition gives the restriction of $\hat{E}^{i}$ to the curves $(X)_{N}$ and $(-X)_{M}$. 
Proposition 1.7. Let $E$ be a coherent sheaf on $X$.

For arbitrary line bundles $N \in \operatorname{Pic}^{-1}(X)$ and $M \in \operatorname{Pic}^{1}(X)$, there are isomorphisms

$$
\begin{aligned}
& \mathrm{FM}_{\mathcal{P}}(E) \otimes^{\mathbf{L}} \mathcal{O}_{(X)_{N}} \cong \mathrm{F}_{+}(E \otimes N) \otimes \mathcal{O}_{X}\left(-P_{0}\right), \\
& \mathrm{FM}_{\mathcal{P}}(E) \otimes^{\mathbf{L}} \mathcal{O}_{(-X)_{M}} \cong \mathrm{F}_{-}(E \otimes M) \otimes \mathcal{O}_{X}\left(P_{0}\right)
\end{aligned}
$$

in the derived category of $(X)_{N} \cong X \cong(-X)_{M}$. Moreover, for general line bundles $N \in \operatorname{Pic}^{-1}(X)$ and $M \in \operatorname{Pic}^{1}(X)$, there are isomorphisms of sheaves

$$
\begin{array}{lll}
\left.\hat{E}^{0}\right|_{(X)_{N}} \cong \mathrm{F}_{+}^{0}(E \otimes N) \otimes \mathcal{O}_{X}\left(-P_{0}\right), & \left.\hat{E}^{1}\right|_{(X)_{N}} \cong \mathrm{F}_{+}^{1}(E \otimes N) \otimes \mathcal{O}_{X}\left(-P_{0}\right) \\
\left.\hat{E}^{0}\right|_{(-X)_{M}} \cong \mathrm{F}_{-}^{0}(E \otimes M) \otimes \mathcal{O}_{X}\left(P_{0}\right), & \left.\hat{E}^{1}\right|_{(-X)_{M}} \cong \mathrm{F}_{-}^{1}(E \otimes M) \otimes \mathcal{O}_{X}\left(P_{0}\right) .
\end{array}
$$

Proof. For the formula for $\operatorname{FM}_{\mathcal{P}}(E) \otimes^{\mathbf{L}} \mathcal{O}_{(X)_{N}}$, we compute

$$
\begin{array}{rlr}
\mathrm{FM}_{\mathcal{P}}(E) \otimes \mathbf{L} \mathcal{O}_{(X)_{N}} & =\operatorname{Rpr}_{P *}\left(\operatorname{pr}_{X}^{*} E \otimes \mathcal{P}\right) \otimes \mathcal{L}_{(X)_{N}} & \text { (definition of } \left.\mathrm{FM}_{\mathcal{P}}\right) \\
& \cong \operatorname{Rpr}_{P *}\left(\operatorname{pr}_{X}^{*} E \otimes \mathcal{P} \otimes \operatorname{pr}_{P}^{*} \mathcal{O}_{(X)_{N}}\right) & \text { (projection formula) } \\
& \cong \operatorname{Rpr}_{P *}\left(\left.\operatorname{pr}_{X}^{*} E \otimes \mathcal{P}\right|_{X \times(X)_{N}}\right) & \text { ( } \mathcal{P} \text { locally free) } \\
& \cong \operatorname{Rpr}_{2 *}\left(\operatorname{pr}_{1}^{*}(E \otimes N) \otimes \mathcal{O}_{X \times X}(\Delta) \otimes \operatorname{pr}_{2}^{*} \mathcal{O}_{X}\left(-P_{0}\right)\right) & (\star) \\
& \cong \operatorname{Rpr}_{2 *}\left(\operatorname{pr}_{1}^{*}(E \otimes N) \otimes \mathcal{O}_{X \times X}(\Delta)\right) \otimes \mathcal{O}_{X}\left(-P_{0}\right) & \text { (projection formula) } \\
& =\mathrm{F}_{+}(E \otimes N) \otimes \mathcal{O}_{X}\left(-P_{0}\right) & \text { (definition of } \left.\mathrm{F}_{+}\right)
\end{array}
$$

where in $(\star)$, we identify $(X)_{N}$ with $X$, so the universal family $\mathcal{P}$ restricted to $X \times(X)_{N}$ is the line bundle $\operatorname{pr}_{1}^{*} N \otimes \mathcal{O}_{X \times X}(\Delta) \otimes \operatorname{pr}_{2}^{*} \mathcal{O}_{X}\left(-P_{0}\right)$ on $X \times X$.

Choosing a nice resolution $0 \rightarrow E_{1} \rightarrow E_{0} \rightarrow E \rightarrow 0$ where the $E_{i}$ are vector bundles with $\mu_{\max }\left(E_{i}\right)<0$, we see that $\operatorname{FM}_{\mathcal{P}}(E)$ can be represented by the complex of vector bundles $\hat{E}_{1}^{1} \rightarrow \hat{E}_{0}^{1}$ on Pic. For a general $N$, the curve $(X)_{N}$ does not contain any of the associated components of the cohomologies of that complex. Thus tensoring with $\mathcal{O}_{(X)_{N}}$ is exact. Similarly for $M$.

\subsection{Orthogonality proofs of semistability of Picard sheaves.}

Proposition 1.8. For a coherent sheaf $E$ on $X$, the following implications hold

$$
(1) \Longleftrightarrow(2) \Longrightarrow(3) \Longleftarrow\left(2^{\prime}\right) \Longleftrightarrow\left(1^{\prime}\right)
$$

among these five conditions:

(1) $(E \otimes M){ }_{-\Delta}^{\perp} F$ for some line bundle $M$ of degree 1 , and a sheaf $F \neq 0$.

(2) For a general line bundle $M$ of degree 1 , the restrictions of the Picard sheaves $\hat{E}^{0}$ and $\hat{E}^{1}$ to the curve $(-X)_{M}$ are both semistable of the same slope.

(3) The sheaves $\hat{E}^{0}$ and $\hat{E}^{1}$ are $\mu$-semistable with respect to the theta divisor on Pic.

$\left(1^{\prime}\right)(E \otimes N) \stackrel{\perp}{\Delta} F$ for some line bundle $N$ of degree -1 , and a sheaf $F \neq 0$.

(2') For a general line bundle $N$ of degree -1, the restrictions of the Picard sheaves $\hat{E}^{0}$ and $\hat{E}^{1}$ to the curve $(X)_{N}$ are both semistable of the same slope.

Proof. The proof of the equivalence $(1) \Longleftrightarrow(2)$ follows from Propositions 1.5 and 1.7, having in mind that twisting with the line bundle $\mathcal{O}_{X}\left(P_{0}\right)$ does not affect semistability. The implication $(2) \Longrightarrow(3)$ is standard: given a sheaf $F$ on a variety $Y$ such that $\left.F\right|_{C}$ is semistable, where $C \subset Y$ is a curve cut out by divisors in the linear system $|H|$ of the polarisation $H$, then $F$ is $\mu_{H^{-}}$-semistable on $Y$ - a destabilising subsheaf $F^{\prime} \subset F$ would induce a destabilising subsheaf $\left.\left.F^{\prime}\right|_{C} \subset F\right|_{C}$.

Remark 1.9. Indeed, the implications $(2) \Longrightarrow(3) \Longleftarrow\left(2^{\prime}\right)$ were also used in [3] as a main tool. Using orthogonality we can not detect whether a semistable sheaf is stable. 


\subsection{A first example.}

Lemma 1.10. Let $E$ be a semistable coherent sheaf on $X$ of degree 0 . Then there exists a coherent sheaf $F \neq 0$ such that $E \frac{\perp}{-\Delta} F$.

Proof. By Faltings' Theorem 1.1, there exists a vector bundle $F$ with $H^{*}(E \otimes F)=0$. Since $F$ can be taken in an open subset in the moduli space of rank $R$ and degree $R(g-1)$ vector bundles, we may furthermore assume that $H^{*}(F)=0$. Tensoring the short exact ideal sheaf sequence of $\Delta$ on $X \times X$ with $E \otimes F=\operatorname{pr}_{1}^{*} E \otimes \operatorname{pr}_{2}^{*} F$, we obtain

$$
\left.0 \rightarrow E \otimes F(-\Delta) \rightarrow E \otimes F \rightarrow E \otimes F\right|_{\Delta} \rightarrow 0 .
$$

We have $H^{*}(E \otimes F)=H^{*}(E) \otimes H^{*}(F)=0$ by the Künneth formula, and $H^{*}\left(\left.E \otimes F\right|_{\Delta}\right)=$ $H^{*}(E \otimes F)=0$ by our assumption. Hence $H^{*}(E \otimes F(-\Delta))=0$, i.e. $E \frac{\perp}{-\Delta} F$.

Corollary 1.11. Let $E$ be a semistable vector bundle on $X$ with $\mu(E)=-1$. Then $\hat{E}^{0}=0$ and $\hat{E}^{1}$ is a vector bundle of rank $g \cdot \operatorname{rk}(E)$ which is also semistable. Moreover, the restriction of $\hat{E}^{1}$ to any curve $(-X)_{M}$ is semistable.

Proof. Since $E$ is semistable of negative degree, we have $\hat{E}^{0}=0$, and it follows that $\hat{E}^{1}$ is a vector bundle of the given rank. Now for any line bundle $M$ of degree 1, the tensor product $E \otimes M$ is semistable of degree 0 . Thus by Lemma 1.10, there exists a sheaf $F \neq 0$ such that $(E \otimes M) \underset{-\Delta}{\perp} F$, and Proposition 1.8 shows the semistability of $\hat{E}^{1}$.

Finally, $\hat{E}^{1}$ is semistable when restricted to any curve $(-X)_{M}$ because the base change argument from the proof of Proposition 1.7 simplifies, with $\operatorname{FM}_{\mathcal{P}}(E)=\hat{E}^{1}[-1]$ a shifted vector bundle.

\subsection{A more subtle example.}

Lemma 1.12. Assume that the genus $g \neq 1$. Let $E$ be a vector bundle on $X$ of slope $\mu(E)=g-1$. Then the following three conditions are equivalent:

(1) $H^{*}(E)=0$.

(2) $E \frac{\perp}{-\Delta} \mathcal{O}_{X}$

(3) There exists a coherent sheaf $F \neq 0$ such that $E \frac{\perp}{-\Delta} F$.

Proof. (1) $\Longrightarrow$ (2): From $H^{*}(E)=0$ we get $H^{*}\left(E \otimes \mathcal{O}_{X}\right)=H^{*}(E) \otimes H^{*}\left(\mathcal{O}_{X}\right)=0$ and $H^{*}\left(\left.E \otimes \mathcal{O}_{X}\right|_{\Delta}\right)=H^{*}(E)=0$. Therefore, the long exact cohomology sequence of $\left.0 \rightarrow E \otimes \mathcal{O}_{X}(-\Delta) \rightarrow E \otimes \mathcal{O}_{X} \rightarrow E \otimes \mathcal{O}_{X}\right|_{\Delta} \rightarrow 0$ gives $\left.E \otimes \mathcal{O}_{X}(-\Delta)\right)=0$, i.e. $E \frac{\perp}{{ }_{-}} \mathcal{O}_{X}$.

$(2) \Longrightarrow(3)$ : This implication is obvious.

$(3) \Longrightarrow(1)$ : If we have such an orthogonality, then $E$ and $F$ are semistable. Note that $\operatorname{deg}(F)=0$ by Lemma 1.2(ii). Moreover, $E$ is also orthogonal to the direct sums $F^{\oplus N}$ for all $N>0$, which are semistable sheaves of $\operatorname{rank} N \cdot \operatorname{rk}(F)$ and degree 0 . We may take $N$ big enough such that the theta divisor $\Theta_{E}$ in the moduli space of semistable degree 0 bundles on $X$ of $\operatorname{rank} N \cdot \operatorname{rk}(F)$ is effective. By semicontinuity, being orthogonal to $E$ is an open condition. Thus, we may assume there exists an $F^{\prime}$ outside $\Theta_{E}$, i.e. $E \stackrel{\perp}{\perp} F^{\prime}$ and $H^{*}\left(E \otimes F^{\prime}\right)=0$. Again we consider the long exact cohomology sequence of $\left.0 \rightarrow E \otimes F^{\prime}(-\Delta) \rightarrow E \otimes F^{\prime} \rightarrow E \otimes F^{\prime}\right|_{\Delta} \rightarrow 0$; here it yields $H^{*}(E) \otimes H^{*}\left(F^{\prime}\right)=0$. We have $\chi\left(F^{\prime}\right) \neq 0$ from Riemann-Roch, if $g \neq 1$. So $H^{*}\left(F^{\prime}\right) \neq 0$, hence $H^{*}(E)=0$.

Corollary 1.13. Let $E$ be a semistable sheaf of slope $g-2$. If there exists a line bundle $M$ of degree 1 with $H^{*}(E \otimes M)=0$, then $\hat{E}^{0}=0$, and $\hat{E}^{1}$ is semistable of rank $\operatorname{rk}(E)$.

Remark 1.14. Raynaud proved in [11] the existence of stable sheaves $E$ having integral slope with the following property: $H^{*}(E \otimes M) \neq 0$ for all line bundles $M$. These base points of the theta divisor form a proper closed subset of the moduli space. Thus, we can only hope that the Picard sheaves $\hat{E}$ are semistable for general semistable sheaves $E$. 


\section{A Classical PROOF FOR the Stability of $\hat{E}$}

We take $E$ to be a globally generated vector bundle on $X$. Thus, we have

$$
0 \rightarrow \mathrm{F}_{-}^{0}(E) \rightarrow H^{0}(E) \otimes \mathcal{O}_{X} \rightarrow E \rightarrow 0 \quad \text { and } \quad \mathrm{F}_{-}^{1}(E) \cong H^{1}(E) \otimes \mathcal{O}_{X} .
$$

Note that a semistable bundle of slope $>2 g-1$ is globally generated.

For a subsheaf $E^{\prime} \subset E$ we obtain an injective map $H^{0}\left(E^{\prime}\right) \hookrightarrow H^{0}(E)$. From both morphisms we eventually obtain a subsheaf $\mathrm{F}_{-}^{0}\left(E^{\prime}\right) \subset \mathrm{F}_{-}^{0}(E)$. The next result tells us, that these subsheaves are enough to test (semi)stability.

Lemma 2.1. The sheaf $\mathrm{F}_{-}^{0}(E)$ is (semi)stable if for all globally generated subsheaves $E^{\prime} \subset E$ we have the inequality $\mu\left(\mathrm{F}_{-}^{0}\left(E^{\prime}\right)\right)(\leq) \mu\left(\mathrm{F}_{-}^{0}(E)\right)$.

Proof. We have the short exact sequence $0 \rightarrow \mathrm{F}_{-}^{0}(E) \rightarrow H^{0}(E) \otimes \mathcal{O}_{X} \rightarrow E \rightarrow 0$, as $E$ is globally generated. Let $U \subset \mathrm{F}_{-}^{0}(E)$ be a subbundle. The inclusion $U \hookrightarrow H^{0}(E) \otimes \mathcal{O}_{X}$ induces a surjection $H^{0}(E)^{\vee} \otimes \mathcal{O}_{X} \rightarrow H^{0}\left(U^{\vee}\right) \otimes \mathcal{O}_{X}$. Denote by $V^{\vee} \subseteq H^{0}\left(U^{\vee}\right)$ the image of the induced map on global sections, $H^{0}(E)^{\vee} \rightarrow H^{0}\left(U^{\vee}\right)$. The commutative diagram

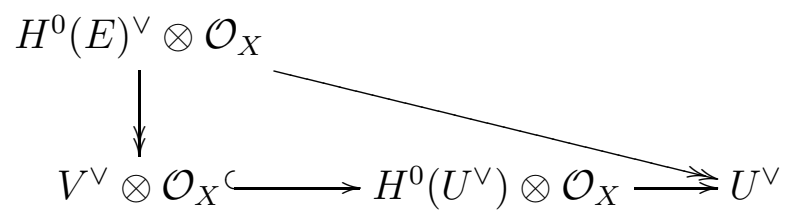

shows that $V^{\vee} \otimes \mathcal{O}_{X} \rightarrow U^{\vee}$ is surjective. We get inclusions $U \hookrightarrow V \otimes \mathcal{O}_{X} \hookrightarrow H^{0}(E) \otimes \mathcal{O}_{X}$, which combine into a commutative diagram of short exact sequences

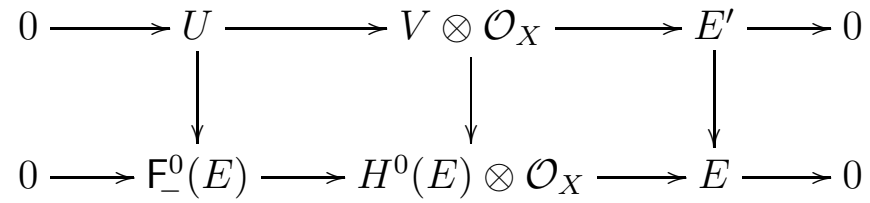

where all vertical arrows are injective. Denote $v=\operatorname{dim}(V)$ and $r^{\prime}=\operatorname{rk}\left(E^{\prime}\right)$. Then

$$
\mu(U)=-\frac{\operatorname{deg}\left(E^{\prime}\right)}{v-r^{\prime}} \leq-\frac{\operatorname{deg}\left(E^{\prime}\right)}{h^{0}\left(E^{\prime}\right)-r^{\prime}}=\mu\left(\mathrm{F}_{-}^{0}\left(E^{\prime}\right)\right)
$$

which shows that testing the (semi)stability condition only on subsheaves of the form $\mathrm{F}_{-}^{0}\left(E^{\prime}\right)$ suffices to deduce it for arbitrary subsheaves.

Corollary 2.2. If $E$ is a (semi)stable vector bundle of slope $\mu(E)>2 g$, then $\mathrm{F}_{-}^{0}(E)$ is (semi)stable.

Proof. Since $\mu:=\mu(E)>2 g-2$, we have $h^{1}(E)=0$ and $h^{0}(E)=\operatorname{deg}(E)+(1-g) \operatorname{rk}(E)$. From the short exact sequence $0 \rightarrow \mathrm{F}_{-}^{0}(E) \rightarrow H^{0}(E) \otimes \mathcal{O}_{X} \rightarrow E \rightarrow 0$, we deduce

$$
\mu\left(\mathrm{F}_{-}^{0}(E)\right)=\frac{-\operatorname{deg}(E)}{h^{0}(E)-\operatorname{rk}(E)}=\frac{-\operatorname{deg}(E)}{\chi(E)-\operatorname{rk}(E)}=\frac{-\operatorname{deg}(E)}{\operatorname{deg}(E)-g \cdot \operatorname{rk}(E)}=\frac{-\mu}{\mu-g} .
$$

Assume that $E$ is stable. Let $E^{\prime} \subsetneq E$ be a globally generated proper subsheaf of $E$. In Corollary 4.2 (Section 4 is independent of the rest of the article), we draw the following consequence from the generalised Clifford theorem: $h^{0}\left(E^{\prime}\right)-\operatorname{rk}\left(E^{\prime}\right)<\frac{\mu-g}{\mu} \operatorname{deg}\left(E^{\prime}\right)$. Thus

$$
\mu\left(\mathrm{F}_{-}^{0}\left(E^{\prime}\right)\right)=\frac{-\operatorname{deg}\left(E^{\prime}\right)}{h^{0}\left(E^{\prime}\right)-\operatorname{rk}\left(E^{\prime}\right)}<\frac{-\mu}{\mu-g}=\mu\left(\mathrm{F}_{-}^{0}(E)\right) .
$$

By Lemma 2.1, to show the stability of $\mathrm{F}_{-}^{0}(E)$ it suffices to check this inequality for the subsheaves of type $\mathrm{F}_{-}^{0}\left(E^{\prime}\right)$. The claim about semistability follows from this by the Jordan-Hölder filtration of $E$. 
Corollary 2.3. Let $E$ be a (semi)stable vector bundle of slope $\mu(E)>2 g-1$. Then the restriction of $\hat{E}^{0}$ to any curve $(-X)_{M}$ is (semi)stable. In particular, $\hat{E}^{0}$ is (semi)stable.

Proof. We have $\left.\hat{E}^{0}\right|_{(-X)_{M}} \cong \mathrm{F}_{-}^{0}(E \otimes M) \otimes \mathcal{O}_{X}\left(P_{0}\right)$ from Proposition 1.7. This holds for all curves $(-X)_{M}$ because $E$ semistable of slope $>2 g-1$ implies that $\hat{E}^{0}$ is locally free. Moreover, $E \otimes M$ is a (semi)stable bundle of slope $\mu(E \otimes M)>2 g$, so Corollary 2.2 applies and yields the (semi)stability of $\mathrm{F}_{-}^{0}(E \otimes M)$ and hence of $\left.\hat{E}^{0}\right|_{(-X)_{M}}$.

\section{Application of orthogonality}

There are two ways how to apply the orthogonality condition $E \frac{\perp}{-\Delta} F$, in order to deduce the semistability of the Picard bundle $\hat{F}$ from the semistability of another Picard sheaf $\hat{F}$. Either we use the symmetry of orthogonality, i.e. Lemma 1.3(i) and (ii), or we employ Serre duality, i.e. Lemma 1.3(iii). We start with the latter method.

Corollary 3.1. Let $E$ be a semistable vector bundle on $X$ with $\mu(E)=2 g-1$. Then $\hat{E}^{1}=0$ and $\hat{E}^{0}$ is a vector bundle of rank $g \cdot \operatorname{rk}(E)$ which is also semistable. Moreover, the restriction of $\hat{E}^{0}$ to any curve $(X)_{N}$ is semistable.

Proof. The vanishing of $\hat{E}^{1}$ follows from cohomology and base change, and for the same reason, $\hat{E}^{0}$ is a vector bundle of the given rank. As $E$ is semistable, so is its dual $E^{\vee}$. Thus, $E^{\vee} \otimes \omega_{X}$ is semistable of degree -1 . By Lemma 1.10, there exists a sheaf $F$ such that $\left(E^{\vee} \otimes \omega_{X} \otimes M\right) \underset{-\Delta}{\perp} F$ for any line bundle $M$ of degree 1. By Serre duality, Lemma 1.3(iii), this implies $\left(E \otimes M^{\vee}\right) \frac{\perp}{\Delta}\left(F^{\vee} \otimes \omega_{X}\right)$. Now we proceed as in the proof of Corollary 1.11.

Remark 3.2. Applying Corollary 3.1 to a degree $2 g-1$ line bundle $L$, we obtain the semistability of the Picard bundle $P_{2 g-1}=\hat{L}^{0}$. Thus, the above result is a generalisation of Kempf's result [6]. In fact, Kempf shows the stability of $P_{2 g-1}$. The stability follows along the lines of Corollaries 2.2 and 4.2. Indeed, if $X$ is not hyperelliptic, and $L \otimes M \otimes \omega_{X}^{\vee}$ is not effective, then the restriction of $\hat{L}^{0}$ to $(-X)_{M}$ is stable.

Lemma 3.3. If $E$ is a stable vector bundle on $X$ with $\mu(E)<-2$, then $\mathrm{F}_{+}^{1}(E)$ is stable.

Proof. Tensoring the short exact sequence $0 \rightarrow \mathcal{O}_{X \times X} \rightarrow \mathcal{O}_{X \times X}(\Delta) \rightarrow \mathcal{O}_{\Delta}(\Delta) \rightarrow 0$ on $X \times X$ with $E \otimes \mathcal{O}_{X}$, and applying $\operatorname{pr}_{2 *}$ yields the following short exact sequence on $X$ :

$$
0 \rightarrow E \otimes \omega^{\vee} \rightarrow H^{1}(E) \otimes \mathcal{O}_{X} \rightarrow \mathrm{F}_{+}^{1}(E) \rightarrow 0,
$$

because $\mathrm{F}_{-}^{0}(E)=0$ from stability of $E$ with $\mu(E)<-2$. Dualising this sequence yields

$$
0 \rightarrow\left(\mathrm{F}_{+}^{1}(E)\right)^{\vee} \rightarrow H^{1}(E)^{\vee} \otimes \mathcal{O}_{X} \rightarrow E^{\vee} \otimes \omega_{X} \rightarrow 0 .
$$

Thus, by classical Serre duality, $\mathrm{F}_{+}^{1}(E)$ is the dual of $\mathrm{F}_{-}^{0}\left(E^{\prime}\right)$ for $E^{\prime}=E^{\vee} \otimes \omega_{X}$. However $E^{\prime}$ is also stable, of slope $\mu\left(E^{\prime}\right)=2 g-2-\mu(E)>2 g$. So by Corollary 2.2 the sheaf $\mathrm{F}_{-}^{0}\left(E^{\prime}\right)$ is stable. This proves the lemma.

Corollary 3.4. If the vector bundle $E$ on $X$ is (semi)stable of slope $\mu(E)<-1$, then the Picard sheaf $\hat{E}^{1}$ is (semi)stable when restricted to any curve $(X)_{N}$. In particular, $\hat{E}^{1}$ is (semi)stable.

Proof. For stability, this follows from Proposition 1.7 and Lemma 3.3.

The claim for semistable $E$ then follows using the Jordan-Hölder filtration of $E$.

Next, we give examples for how to apply the symmetry property of orthogonality. As usual, for a rational number $x$ we denote by $\lceil x\rceil$ the round up of $x$. For any $r, h \in \mathbb{N}$, 
we introduce the number used by Popa in [10, Theorem 5.3] for an effective version of Faltings' Theorem 1.1:

$$
P(r, h):=2 h\left\lceil\frac{h^{2} r^{2}+1}{8 h}\right\rceil \text {. }
$$

Theorem 3.5 (Popa 2001). For any semistable vector bundle $G$ of rank $r \cdot h$ and degree $d \cdot h$ with coprime integers $r$ and $d$, there exists for any $k \geq P(r, h)$ a vector bundle $F$ of rank $r \cdot k$ such that $H^{*}(G \otimes F)=0$.

Lemma 3.6. Let $\mu=\frac{d}{r} \in \mathbb{Q}$ with $\mu \in(g, g+1]$, let $k \geq P(r, g)$ and $R=k \cdot r$. Then there exists a vector bundle $F$ on $X$ of slope $\mu$ and rank $R$ such that $\mathrm{F}_{-}^{0}(F) \oplus \mathrm{F}_{-}^{1}(F)$ is semistable.

Proof. We begin with the involution $\mathbb{Q} \rightarrow \mathbb{Q}, \mu \mapsto \mu^{-}:=g+\frac{g}{\mu-g}$. It is decreasing on $\mathbb{Q}_{>g}$. By Lemma 1.2, if $E \frac{\perp}{-\Delta} F$, then $\mu(E)=\mu(F)^{-}$.

Now let $\mu=\frac{d}{r} \in(g, g+1]$, then $\mu^{-} \in[2 g, \infty)$. Let $E$ be a stable vector bundle on $X$ of rank $d-r g$ and degree $g d-g^{2} r+r g$, i.e. $\mu(E)=\mu^{-}$. By Corollary 2.2 or Remark 3.2, we have that $\mathrm{F}_{-}^{0}(E)$ is semistable. Since $\mu(E)>2 g-1$, we also conclude $\mathrm{F}_{-}^{1}(E)=0$. So we can use the Riemann-Roch formula to compute $\operatorname{rk}_{(}\left(\mathrm{F}_{-}^{0}(E)\right)=g r$, and $\operatorname{deg}\left(\mathrm{F}_{-}^{0}(E)\right)=g r-g d-g^{2} r$. Popa's result Theorem 3.5 implies that for any $R=k \cdot r$ with $k$ as above there exists a vector bundle $F$ of $\operatorname{rank} R$ such that $H^{*}\left(\mathrm{~F}_{-}^{0}(E) \otimes F\right)=0$.

By Lemma 1.4, this yields $E \frac{\perp}{-\Delta} F$. Symmetry, i.e. Lemma 1.3(i), then gives $F \frac{\perp}{-\Delta} E$. And so, again by Lemma 1.4, it follows that $H^{*}\left(\left(\mathrm{~F}_{-}^{0}(F) \oplus \mathrm{F}_{-}^{1}(F)\right) \otimes E\right)=0$. This implies the semistability of the direct $\operatorname{sum} \mathrm{F}_{-}^{0}(F) \oplus \mathrm{F}_{-}^{1}(F)$.

Proposition 3.7. Let $\mu=\frac{d}{r} \in \mathbb{Q}$ with $\mu \in(g-1, g]$, let $k \geq P(r, g)$ and $R=k \cdot r$. Then there exists a vector bundle $F$ on $X$ of slope $\mu$ and rank $R$ such that $\hat{F}^{0}$ is semistable. Indeed, the restriction of $\hat{F}^{0}$ to the general curve $(-X)_{M}$ is semistable.

Proof. It is enough to show the existence of some $F$ such that $\hat{F}^{0}$ restricted to $(-X)_{M}$ is semistable. Let $\mu$ and $R$ be as in the proposition. Let $F^{\prime}$ be a vector bundle of slope $\mu+1$ and rank $R$ such that $\mathrm{F}_{-}^{0}(F) \oplus \mathrm{F}_{-}^{1}(F)$ is semistable, which exists by Lemma 3.6. Then $\mathrm{F}_{-}^{0}\left(F^{\prime}\right) \otimes \mathcal{O}_{X}\left(P_{0}\right)$ is also semistable.

We set $F=F^{\prime} \otimes M^{\vee}$ for some line bundle $M$ of degree 1. By Proposition 1.7, the restriction of $\hat{F}^{0}$ to $(-X)_{M}$ is the semistable sheaf $F_{-}^{0}\left(F^{\prime}\right) \otimes \mathcal{O}_{X}\left(P_{0}\right)$.

Corollary 3.8. Let $\mu=\frac{d}{r} \in \mathbb{Q}$ with $\mu \in[g-2, g-1)$, let $k \geq P(r, g)$ and $R=k \cdot r$. Then there exists a vector bundle $F$ on $X$ of slope $\mu$ and rank $R$ such that $\hat{F}^{1}$ is semistable. Indeed, the restriction of $\hat{F}^{1}$ to the general curve $(X)_{N}$ is semistable.

Proof. This follows from Proposition 3.7, using Serre duality as in Lemma 1.3(iii).

\section{Clifford's theorem for Vector Bundles on A CURVE}

Let us remind the reader that $\mu_{\max }(E)$ denotes the maximum of all slopes of subbundles of $E$, and $\mu_{\min }(E)$ denotes the minimal slope of all quotient bundles of $E$.

Proposition 4.1. Let $E$ be a vector bundle of rank $r$ and degree $d$ on the smooth projective curve $X$ of genus $g$. If $\mu_{\max }(E) \leq 2 g-2$ and $\mu_{\min }(E) \geq 0$, then we have the estimate

$$
h^{0}(E)-r \leq \frac{d}{2} .
$$

Moreover, if $\mu_{\max }(E)<2 g-2$ and $\mu_{\min }(E)>0$ and $h^{0}(E)-r=\frac{d}{2}$, then $X$ is hyperelliptic, and the determinant line bundle $\operatorname{det}(E)$ is a multiple of the hyperelliptic line bundle $M$, and $E$ possesses a filtration with graded object $\operatorname{gr}(E)=\bigoplus_{i=1}^{r} M^{\otimes a_{i}}$ with $0<a_{i}<g-1$. 
Proof. We first prove the inequality $h^{0}(E)-r \leq \frac{d}{2}$ by induction on $r$.

If $r=1$, then $E=\mathcal{O}(D)$ is a line bundle associated to a divisor $D$. In this case $d=\mu_{\max }(\mathcal{O}(D))=\mu_{\min }(\mathcal{O}(D))$. If $D$ is effective and special, then the claim is precisely the well known theorem of Clifford, see for example [5, Theorem IV.5.4]. If $D$ is not effective, then $h^{0}(\mathcal{O}(D))=0$ and the statement is trivial. If $D$ is non-special, then by Riemann-Roch $h^{0}(\mathcal{O}(D))-1=\chi(\mathcal{O}(D))-1=d-g<\frac{d}{2}$, the inequality following from $d=\mu_{\max } \leq 2 g-2$.

Now suppose that $E$ is of rank $r \geq 2$, and the inequality holds for all vector bundles of rank smaller than $r$ which meet the slope conditions. We consider two cases:

Case 1: $E$ is not semistable. Take the subsheaf $E_{1}$ of $E$ of slope $\mu_{\max }(E)$ and of maximal possible rank. This $E_{1}$ is the first sheaf appearing in the Harder-Narasimhan filtration of $E$. We obtain a short exact sequence

$$
0 \rightarrow E_{1} \rightarrow E \rightarrow E_{2} \rightarrow 0 \text {. }
$$

We have $\mu_{\max }\left(E_{1}\right)=\mu_{\min }\left(E_{1}\right)=\mu_{\max }(E), \mu_{\max }(E)>\mu_{\max }\left(E_{2}\right)$, and $\mu_{\min }(E)=\mu_{\min }\left(E_{2}\right)$. In particular, we see that the induction hypothesis applies to the vector bundles $E_{1}$ and $E_{2}$. Hence $h^{0}\left(E_{i}\right)-\operatorname{rk}\left(E_{i}\right) \leq \frac{1}{2} \operatorname{deg}\left(E_{i}\right)$ for $i \in\{1,2\}$. Taking global sections of the above short exact sequence, we conclude $h^{0}(E) \leq h^{0}\left(E_{1}\right)+h^{0}\left(E_{2}\right)$. So we get

$$
\begin{aligned}
h^{0}(E)-r & \leq h^{0}\left(E_{1}\right)+h^{0}\left(E_{2}\right)-r=\left(h^{0}\left(E_{1}\right)-\operatorname{rk}\left(E_{1}\right)\right)+\left(h^{0}\left(E_{2}\right)-\operatorname{rk}\left(E_{2}\right)\right) \\
& \leq \frac{1}{2} \operatorname{deg}\left(E_{1}\right)+\frac{1}{2} \operatorname{deg}\left(E_{2}\right)=\frac{1}{2} \operatorname{deg}(E) .
\end{aligned}
$$

Case 2: $E$ is semistable. Again, we distinguish two cases, by inspecting the slope of $E$. Case 2.1: $\mu(E) \leq g-1$. We may assume $h^{0}(E)>0$. Let $E_{1}$ be a line subbundle of $E$ of maximal possible degree $d_{1}$. From $h^{0}(E)>0$ we conclude that $d_{1} \geq 0$. We obtain a short exact sequence

$$
0 \rightarrow E_{1} \rightarrow E \rightarrow E_{2} \rightarrow 0
$$

Since any quotient of $E_{2}$ is also a quotient of $E$ we conclude $\mu_{\min }\left(E_{2}\right) \geq \mu_{\min }(E) \geq 0$. We want to show that $\mu_{\max }\left(E_{2}\right) \leq 2 g-2$. Assume the contrary. Then we have a subsheaf $E_{3} \subset E_{2}$ of rank $r_{3}$ and slope $\mu\left(E_{3}\right)>2 g-2$. The kernel $K$ of the composition of surjections

$$
E \rightarrow E_{2} \rightarrow E_{2} / E_{3}
$$

is of rank $r_{3}+1$ and of slope $\mu(K)=\frac{d_{1}+\mu\left(E_{3}\right) \cdot r_{3}}{r_{3}+1} \geq \frac{\mu\left(E_{3}\right) \cdot r_{3}}{r_{3}+1}=\frac{\mu\left(E_{3}\right)}{1+1 / r_{3}}>\frac{2 g-2}{1+1 / r_{3}} \geq g-1$. This contradicts the semistability of $E$. Thus, for both sheaves $E_{1}$ and $E_{2}$ the induction hypothesis applies, and we can proceed like in Case 1.

Case 2.2: $\mu\left(E_{1}\right)>g-1$. The Serre dual bundle $E^{\prime}=E^{\vee} \otimes \omega_{X}$ has slope $\mu\left(E^{\prime}\right)=$ $2 g-2-\mu(E)<g-1$. Therefore, as we have seen in Case 2.1

$$
h^{0}\left(E^{\prime}\right) \leq \frac{\operatorname{deg}\left(E^{\prime}\right)}{2}=\frac{\operatorname{rk}(E)(2 g-2)-\operatorname{deg}(E)}{2} .
$$

By Serre duality $h^{0}\left(E^{\prime}\right)=h^{1}(E)$. So when adding the Riemann-Roch formula $h^{0}(E)-$ $h^{1}(E)=\operatorname{deg}(E)+\operatorname{rk}(E)(1-g)$ to the above inequality, we obtain the stated inequality.

The statement for the case $h^{0}(E)-r=\frac{d}{2}$ follows along the same lines. Indeed, we must have this equality for $E_{1}$ and $E_{2}$ and can proceed by induction since $\operatorname{det}(E) \cong$ $\operatorname{det}\left(E_{1}\right) \otimes \operatorname{det}\left(E_{2}\right)$. The passage from $E$ to the Serre dual $E^{\prime}=E^{\vee} \otimes \omega_{X}$ sends a vector bundle $E$ with $\operatorname{det}(E)=M^{\otimes a}$ to a bundle with $\operatorname{det}\left(E^{\prime}\right)=M^{\otimes(r(g-1)-a)}$ where $M$ denotes the hyperelliptic line bundle. 
Corollary 4.2. Let $E$ be a stable vector bundle of slope $\mu=\mu(E)>2 g$. For any globally generated subsheaf $E^{\prime} \subsetneq E$ which is not a trivial bundle, we have the strict inequality

$$
h^{0}\left(E^{\prime}\right)-\operatorname{rk}\left(E^{\prime}\right)<\left(1-\frac{g}{\mu}\right) \operatorname{deg}\left(E^{\prime}\right) .
$$

Proof. As $E^{\prime} \subset E$ is globally generated, we have $\mu_{\min }\left(E^{\prime}\right) \geq 0$. For one sheaf $E_{1}^{\prime}$ in the Harder-Narasimhan filtration of $E^{\prime}$, we have $\mu_{\min }\left(E_{1}^{\prime}\right)>2 g-2$, and $\mu_{\max }\left(E^{\prime} / E_{1}^{\prime}\right) \leq 2 g-2$. We set $E_{2}^{\prime}=E^{\prime} / E_{1}^{\prime}$. Now $E_{1}^{\prime}$ is semistable with $\mu_{\min }\left(E_{1}^{\prime}\right)>2 g-2$, hence $h^{1}\left(E_{1}^{\prime}\right)=0$. So

$$
h^{0}\left(E_{1}^{\prime}\right)-\operatorname{rk}\left(E_{1}^{\prime}\right)=\chi\left(E_{1}^{\prime}\right)-\operatorname{rk}\left(E_{1}^{\prime}\right)=\operatorname{deg}\left(E_{1}^{\prime}\right)-g \cdot \operatorname{rk}\left(E_{1}^{\prime}\right)=\left(1-\frac{g}{\mu\left(E_{1}^{\prime}\right)}\right) \operatorname{deg}\left(E_{1}^{\prime}\right)
$$

by Riemann-Roch. Since $\mu\left(E_{1}^{\prime}\right)<\mu$, and the function $x \mapsto 1-\frac{g}{x}$ is strictly increasing for $x>0$, we deduce the inequality

$$
h^{0}\left(E_{1}^{\prime}\right)-\operatorname{rk}\left(E_{1}^{\prime}\right)<\left(1-\frac{g}{\mu}\right) \operatorname{deg}\left(E_{1}^{\prime}\right)
$$

The sheaf $E_{2}^{\prime}$ satisfies the assumptions of Proposition 4.1. Moreover, $E_{2}^{\prime}$ is itself a globally generated sheaf, and we have $\mu_{\min }\left(E_{2}^{\prime}\right) \geq \mu_{\min }\left(E^{\prime}\right)>0$, the latter inequality from the assumption that $E^{\prime}$ is not a trivial bundle. Hence $\operatorname{deg}\left(E_{2}^{\prime}\right)>0$ and so we have

$$
h^{0}\left(E_{2}^{\prime}\right)-\operatorname{rk}\left(E_{2}^{\prime}\right) \leq \frac{1}{2} \operatorname{deg}\left(E_{2}^{\prime}\right)<\left(1-\frac{g}{\mu}\right) \operatorname{deg}\left(E_{2}^{\prime}\right) .
$$

This last inequality holds, because $\mu>2 g$ implies $\frac{1}{2}<1-g / \mu$. Adding the two inequalities for $h^{0}\left(E_{i}^{\prime}\right)-\operatorname{rk}\left(E_{i}^{\prime}\right)$ for $i=1,2$, we obtain the statement of the corollary.

\section{REFERENCES}

[1] I. Biswas, L. Brambila-Paz, T.L. Gómez, P.E. Newstead: Stability of the Picard bundle, Bull. London Math. Soc. 34 (2002), 561-568.

[2] L. Brambila-Paz, I. Grzegorczyk, P. Newstead: Geography of Brill-Noether loci for small slopes, J. Alg. Geom. 6 (1997), 645-669, also arXiv:alg-geom/9511003.

[3] L. Ein, R. Lazarsfeld: Stability and restrictions of Picard bundles, with an application to the normal bundles of elliptic curves, Complex projective geometry (Trieste, 1989/Bergen, 1989), 149-156, London Math. Soc. Lecture Note Ser., 179, Cambridge Univ. Press, Cambridge, 1992.

[4] G. Faltings: Stable G-bundles and projective connections, J. Alg. Geom. 2 (1993), 507-568.

[5] R. Hartshorne: Algebraic Geometry, GTM 52, Springer New York, 1977.

[6] G. Kempf: Rank g Picard bundles are stable, Amer. J. Math. 112 (1990), 397-401.

[7] Y. Li: Spectral curves, theta divisors and Picard bundles, Internat. J. Math. 2 (1991), 525-550.

[8] A. Mattuck: Picard bundles, Illinois J. Math. 5 (1961), 550-564.

[9] S. Mukai: Duality between $D(X)$ and $D(\hat{X})$ with its application to Picard sheaves, Nagoya Math. J. 81 (1981), 153-175.

[10] M. Popa: Dimension estimates for Hilbert schemes and effective base point freeness on moduli spaces of vector bundles on curves, Duke Math J. 107, (2001), 469-495, also arXiv:math/0002018.

[11] M. Raynaud: Sections des fibrés vectoriels sur une courbe, Bull. Soc. Math. France 110 (1982), $103-125$.

[12] R.L.E. Schwarzenberger: Jacobians and symmetric products, Illinois J. Math. 7 (1963), 257-268.

Email: georg.hein@uni-due.de, ploog@math.uni-hannover.de 